\title{
Absolute cardiovascular disease risk and the use of the Australian cardiovascular disease risk calculator
}

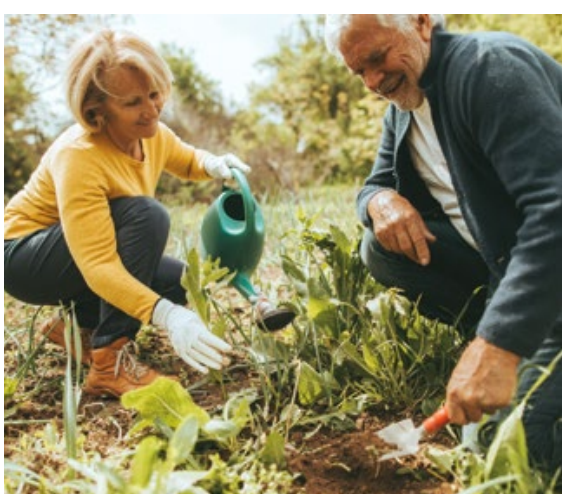

CPD

\section{Mark Nelson}

\section{Background}

Primary prevention of cardiovascular disease (CVD) has been traditionally guided by individual risk factors such hypertension and hypercholesterolaemia. An absolute risk-based approach is more effective.

\section{Objective}

The aim of this article is to outline the superiority of an absolute risk-based approach when compared with individual risk factor management for the primary prevention of CVD, and to elaborate on the derivation and use of the Australian absolute CVD risk calculator.

\section{Discussion}

An absolute risk-based approach is superior to the traditional individual risk factor approach when identifying which patients would benefit most from the prescription of blood pressure-lowering and lipid-lowering medications.
CASE

John, a smoker aged 61 years, presented for prescriptions post-hospital discharge after his first inferior myocardial infarction. The general practitioner (GP) examined John's cardiovascular system, supplied him with prescriptions for medications that had been initiated during his hospital stay, and reinforced his need to attend cardiac rehabilitation. The GP revisited John's file at lunchtime to conduct a critical event audit of the two years prior to his event. His cardiovascular risk factors had been previously assessed, but he had never been given any blood pressure-lowering or lipid-lowering medication because these values were in the 'normal' range. The GP entered John's pre-event risk factors into the Australian cardiovascular risk calculator in the clinical software and the result appeared in red (high risk 17\%).

John's case is a real indication of the problems of managing blood pressure and lipids as individual cardiovascular risk factors rather than considering the patient holistically. John had the two most important risk factors for adverse risk - older age and male sex - and the most important modifiable risk factor: he was a smoker. In this case study, John's myocardial infarction was not prevented as he had not been considered to be at risk because his blood pressure and lipid levels were in the 'normal' range. This is not uncommon, with MedicineInsight data suggesting that only $7 \%$ of the population aged 45-74 years attending Australian general practice have had a recent absolute risk score calculated. ${ }^{1}$

\section{Absolute risk}

Absolute risk is the risk of having an event over a specified period, usually five or 10 years. The algorithms that score individuals only include the best predictive factors to aid ease of use. Most of the world uses 10 years as the time period. Australia and New Zealand have chosen five years as this aligns with the length of clinical trials from which the evidence of therapeutic benefit is derived and acknowledges discounting, where people give precedence to intermediate-term over long-term outcomes.

Derivation and use of the Australian cardiovascular risk calculator The Australian cardiovascular risk calculator is based on the Framingham Risk Equation recalibrated for the Australian population. ${ }^{2}$ The Framingham Heart Study commenced in 1948 in Framingham, Massachusetts, and is now on its fourth generation. It initially lacked ethnic and age diversity but was groundbreaking 
and timely as it preceded blood pressurelowering and lipid-lowering therapies. The variables identified in the study have survived the rigors of time and are found to be determinants of risk in many populations around the globe. ${ }^{3}$ Ideally, Australia should have a cardiovascular disease (CVD) risk score model for patients based on a large contemporary representative Australian cohort study including information on all CVD risk factors and accumulating sufficient CVD events. ${ }^{4}$ As this is not the case, the recommendation is to continue the use of the current Australian absolute CVD calculator (www.cvdcheck.org.au). The advantage of this approach for therapeutic intervention is that it avoids medicalising low-risk individuals with the costs to the individual and society of medications and monitoring, while intervening for those at high risk who may not cross individual risk factor treatment thresholds, such as John.

\section{Aboriginal and Torres Strait Islander peoples}

Aboriginal and Torres Strait islander peoples have a much higher risk of adverse CVD events, which also tend to occur at an earlier age than in the non-Indigenous population. ${ }^{5}$ Therefore, screening is recommended from the age of 35 years in Aboriginal and Torres Strait islander peoples, 10 years earlier than in the general population. However, a recalibration of the absolute risk score here is elusive, probably because of the considerable heterogeneity in Aboriginal and Torres Strait Islander communities across Australia. For this reason, an absolute risk score should only be seen as a minimal estimation for Aboriginal and Torres Strait Islander peoples; that is, the true risk is likely to be higher, and therefore therapeutic intervention should occur at 'moderate risk' or possibly even lower. ${ }^{6}$

\section{How and when to carry out} the absolute risk assessment Using the Australian absolute CVD risk calculator is now a relatively simple task as most clinical software incorporates it as an icon. The guidelines recommend two-yearly reassessments, ${ }^{2}$ but this recommendation is consensus-based rather than evidence-based, and based on prior individual risk factor screening regimens. Since the guidelines were published, some newer evidence indicates that, on average, it takes approximately a decade before someone is likely to be reclassified; however, this will depend on how close the initial score is to classification thresholds. ${ }^{7}$ Fasting lipids from up to three years prior can be used. ${ }^{8}$ The Heart Health Check (Medicare Benefits Schedule item $699 / 177$ ) has a mandatory calculation of an absolute risk score.

\section{Reclassification}

An absolute risk score gives a good and reliable estimation for most but not all individuals. This is accounted for in the guidelines by the ability to reclassify 'moderate-risk' individuals to a higher risk category and hence to mandate lipid-lowering and blood pressurelowering therapy. ${ }^{6}$ Thus, individuals from higher-risk populations (eg Aboriginal and Torres Strait islander peoples, people of South Asian descent) or those with known additional CVD risk factors (eg a strong family history or morbid obesity) may warrant treatment at lower thresholds ('moderate risk'). This is where additional tests such as calcium scoring may also be useful.

\section{Communicating risk}

There is extensive literature on risk communication. ${ }^{9}$ This is a very important part of the consultation as an asymptomatic person is being asked to take medications lifelong that may have adverse effects, which is likely to change the patients' perception of their own health. Patients are the final arbiter of whether they take their medication or not, as witnessed by the "prescription in the bin' phenomenon. ${ }^{10}$ Charts exist to aid communication of the potential benefits to patients (eg https://bpac.org.nz/ BPJ/2014/September/cvrisk.aspx).

\section{Pharmacotherapy}

When a patient is identified as high risk, both lipid-lowering and blood pressurelowering medications are indicated irrespective of the individual level of the risk factors and subject to tolerability. When a patient is at moderate risk, medication therapy is considered for those who may be reclassified as a result of additional important risk factors. For low-risk individuals, medication is not recommended. Management is universally lifestyle based.

\section{Common criticisms of the absolute risk approach \\ 'My younger patients have more years of disability and life to lose than my older patients.'}

A criticism of the absolute risk score is that it is largely determined by age. This is a valid observation but can also be seen as ageist. Attempts to mitigate the effects of age, such as determining 'whole of life' risk, are hampered by competing causes of premature death and the uncertainty of predicting 50 years into the future. Witness the $75 \%$ population reduction in CVD event rates in the past 50 years. ${ }^{11}$ Who would have predicted that in the 1960s? In younger patients, raised blood pressure is more likely to be driven by adverse lifestyle factors or be secondary to other conditions. Addressing these is paramount, as these behaviours are likely to have other adverse effects, and the underlying condition needs to be treated.

It may be beneficial for different thresholds to be used at different ages, as the thresholds for treatment for absolute risk are as arbitrary as individual risk factors, and cost effectiveness will vary between workforce and retirement ages.

\section{'If my patients live long enough they will all be on drug therapy using this calculator.'}

Most individuals aged $>70$ years are at high risk. Blood pressure-lowering and lipid lowering-medications have been shown to be effective and well tolerated in this age group. Lowering blood pressure in patients who are elderly saves lives and reduces adverse event rates. ${ }^{12,13}$ 


\section{'Why are important risk factors such as family history and obesity omitted?'}

There are more than 250 independent risk factors for CVD. The most accurate estimation of risk therefore would include all or most of these. However, this is an exercise in diminishing returns, as gains are marginal beyond the 'traditional' factors of age, sex, smoking and diabetes status, blood pressure and cholesterol. Family history doubles the CVD risk yet it 'falls out' of the risk algorithm as being one of the better predictors. Why? There are probably three reasons. First, family history is not a genetic history. Environmental factors are at play. If a patient's parents smoked, the patient is more likely to smoke, and therefore part of the 'family history' is offset as personal smoking history. This is also likely to be seen in dietary exposure manifesting as higher blood pressure and cholesterol. Second, family history is unreliable as it is often based on hearsay rather than medical records. A patient-reported paternal 'heart attack' at the age of 60 years may have been an isolated episode of atrial fibrillation. If you have reliable knowledge of an adverse premature family history, then this can be used to reclassify an individual as mentioned previously. Third, cause of death is subject to probabilistic attribution. As CVD is one of the major causes of death, it often is entered on death certificates in circumstances where the cause is unclear.

\section{Conclusion}

All patients aged 45-74 years should have a contemporary absolute risk score in their history much as they have a blood pressure reading recorded. Whatever accuracy is lacking in a risk-based approached to therapeutics for the primary prevention of CVD, as a ranking exercise it is superior to previous individual risk factor approaches. It is the logical way to prevent overdiagnosis and overtreatment while giving therapeutics to those who are most likely to benefit from them.
Competing interests: None.

Funding: None.

Provenance and peer review: Commissioned, externally peer reviewed.

\section{References}

1. Raffoul N. CVD risk assessment in Australian primary care. South Australia: GP19 conference, October 2019.

2. National Vascular Disease Prevention Alliance. Guidelines for the assessment of absolute cardiovascular disease risk. Melbourne, Vic: National Stroke Foundation, 2012.

3. Yusuf S, Hawken S, Ounpuu S, et al. Effect of potentially modifiable risk factors associated with myocardial infarction in 52 countries (the INTERHEART study): Case-control study. Lancet 2004;364(9438):937-52. doi: 10.1016/S01406736(04)17018-9.

4. Nelson MR, Woodward M. Developing cardiovascular risk prediction models for Austraia. Med J Aust 2019; 210(4):158-59. doi: 10.5694/ mja2.50010.

5. Calabria B, Korda RJ, Lovett RW, et al. Absolute cardiovascular disease risk and lipid-lowering therapy among Aboriginal and Torres Strait Islander Australians. Med J Aust 2018;209(1):35-41. doi: 10.5694/mja17.00897.

6. National Vascular Disease Prevention Alliance. Guidelines for the management of absolute cardiovascular disease risk. Melbourne, Vic: National Stroke Foundation, 2012.

7. Bell KJ, Hayen A, Irwig L, Takahashi O, Ohde S, Glasziou P. When to remeasure cardiovascular risk in untreated people at low and intermediate risk: Observational study. BMJ 2013;346:f1895. doi: 10.1136/bmj.f1895.

8. Glasziou PP, Irwig L, Heritier S, Simes RJ, Tonkin A. Monitoring cholesterol levels: Measurement error or true change. Ann Inter Med 2008;148(9):656-61. doi: 10.7326/0003-4819-1489-200805060-00005

9. Hill S, Spink J, Cadilhac D, et al. Absolute risk representation in cardiovascular disease prevention: comprehension and preferences of health care consumers and general practitioners involved in a focus group study. BMC Public Health 2010;10:108. doi: 10.1186/1471-2458-10-108.

10. Simons LA, Ortiz M, Calcino G. Long term persistence with statin therapy - Experience in Australia 2006-2010. Aust Fam Physician 2011;40(5):319-22.

11. Australian Institute of Health and Welfare. Trends in cardiovascular deaths. Canberra, ACT: AlHW, 2017. Available at www.aihw.gov.au/getmedia/2ba74f7fd812-4539-a006-ca39b34d8120/aihw-21213.pdf [Accessed 30 June 2020].

12. Wright JT Jr, Williamson JD, Whelton PK, et al. A randomized trial of intensive versus standard blood-pressure control. N Engl J Med 2015;373(22):2103-16. doi: 10.1056/ NEJMoa1511939.

13. Beckett NS, Peters R, Fletcher AE, et al. Treatment of hypertension in patients 80 years of age or older. N Engl J Med 2008;358(18):1887-98. doi: 10.1056/NEJMoa0801369.

\section{Author}

